\title{
Radiation dose in pneumatic reduction of ileo-colic intussusceptions - results from a single-institution study
}

\author{
Jennifer L. Cullmann • Johannes T. Heverhagen • Stefan Puig
}

Received: 13 February 2014 /Revised: 22 August 2014 / Accepted: 22 October 2014 / Published online: 23 November 2014

(C) Springer-Verlag Berlin Heidelberg 2014

\begin{abstract}
Background Air enema under fluoroscopy is a well-accepted procedure for the treatment of childhood intussusception. However, the reported radiation doses of pneumatic reduction with conventional fluoroscopy units have been high in decades past. Objective To compare current radiation doses at our institution to past doses reported by others for fluoroscopic-guided pneumatic reduction of ileo-colic intussusception in children. Materials and Methods Since 2007 radiologists and residents in our department who perform reduction of intussusceptions have received a radiation risk training. We retrospectively analyzed the data of 45 children ( 5 months -8 years) who underwent a total of 48 pneumatic reductions of ileo-colic intussusception between 2008 and 2012. We analyzed data for screening time and dose area product (DAP) and compared these data to those reported up to and including the year 2000. Results Our mean screening time measured by the DAP-meter was $53.8 \mathrm{~s}$ (range $1-320 \mathrm{~s}$, median $33.0 \mathrm{~s}$ ). The mean DAP was $11.4 \mathrm{cGy} \mathrm{cm} \mathrm{cm}^{2}$ (range $1-145 \mathrm{cGy} \mathrm{cm}^{2}$, median $5.45 \mathrm{cGy} \mathrm{cm}^{2}$ ). There was one bowel perforation, in a 1-year-old boy requiring surgical revision. Only three studies in the literature presented radiation exposure results on children who received pneumatic or hydrostatic reduction of intussusception under fluoroscopy. Screening times and dose area products in those studies, which were published in the 1990s and in the year 2000, were substantially higher than those in our sample.

Conclusion Low-frequency pulsed fluoroscopy and other dose-saving keys as well as the radiation risk training might have helped to improve the quality of the procedure in terms of radiation exposure.
\end{abstract}

Keywords Radiation dose · Intussusception · Air enema . Pulsed fluoroscopy $\cdot$ Children

J. L. Cullmann $(\varangle) \cdot J$. T. Heverhagen $\cdot$ S. Puig

Institute for Diagnostic, Interventional, and Pediatric Radiology,

Inselspital, University Hospital Bern,

Freiburgstr. 10, 3010 Bern, Switzerland

e-mail: jennifer.cullmann@insel.ch

\section{Introduction}

Air enema under fluoroscopy is a well-accepted procedure for the diagnosis and treatment of childhood intussusception at many institutions [1-3]. However, the reported radiation doses of pneumatic reduction with conventional non-pulsed fluoroscopy units have been high. For example, one study of 153 pneumatic reductions, published in 2000 , reported a mean screening time of about 6 min and a mean dose area product (DAP) of $366.5 \mathrm{cGy} \mathrm{cm}^{2}$ [3]. In that study, the longest screening time leading to a successful reduction was $21 \mathrm{~min}$. This gave a DAP of $1,278 \mathrm{cGy} \mathrm{cm}^{2}$ and an effective dose of $12.73 \mathrm{mSv}$ in one child [3], equivalent to approximately 400 abdominal radiographs in a 1-year-old.

Several technical innovations, e.g., pulsed fluoroscopy, additional filtration by copper 0.1 or $0.2 \mathrm{~mm}$, and optimized digital image techniques (last image hold, frame grabber), have led to reduced patient radiation dose in more recent years. Additionally, the continuously improving knowledge and ensuing training of radiologists about radiation risks might have resulted in a more efficient control of fluoroscopic time. Therefore, we checked whether radiation doses in childhood intussusception are lower than reported in decades past.

\section{Materials and methods}

Since 2007 radiologists and residents in our department who perform reductions of intussusceptions have received radiation risk training at our pediatric fluoroscopy unit; this training has included patient positioning, tight collimation, use of gonadal capsules in boys, and low-frequency pulsed fluoroscopy. Additionally, our department has established an algorithm for diagnosis and treatment of intussusception that was derived from published findings and made available to the radiologists. For management of complications during the reduction maneuver, such as perforation or non-reducibility, all procedures should, by our department's standard, be performed in the presence of an experienced pediatric surgeon. 
All examinations and interventional procedures are to be supervised by an experienced pediatric radiologist. If performed after hours by a resident, the procedures have to be supervised by a radiologist on-call and discussed with a pediatric radiologist the next day.

We reviewed our clinical records and found that 45 children ( 30 boys and 15 girls) ages 5 months to 8 years underwent a total of 48 pneumatic reductions of an ileo-colic intussusception during the 4-year period of 2008-2012. Three children, ages 1 year, 1 year and 3 years, underwent a recurrent intussusception within $1 \mathrm{~h}, 4 \mathrm{~h}$ and $7 \mathrm{~h}$, respectively.

We analyzed these data for screening time and dose area product (DAP), which is an indicator of the effective radiation dose. Our institutional review board approved the study and waived informed consent.

The 48 procedures were performed by 23 physicians who had 4-20 years of clinical experience. Diagnosis of intussusception was established by sonography with a 9- to $18-\mathrm{MHz}$ linear transducer (Acuson Sequoia or Acuson S2000; Siemens Medical Solutions, Malvern, PA). All reductions were performed in the radiology department and sedation was routinely administered [4]. The children were placed in a prone position. Fluoroscopic monitoring of the reduction procedure was performed with a Diagnost 97 unit (Philips, Eindhoven, The Netherlands) with an overcoach X-ray tube using pulsed fluoroscopy option (1.56 images/s). Images were acquired using additional filtration by copper $0.2 \mathrm{~mm}$, an image intensifier, a fixed grid of 36 lines per centimeter, and last image hold. The screening times and DAP were measured during the procedure with a DAP-meter (Diamentor M4; PTW, Freiburg, Germany).
The results of this study with regard to radiation exposure as well as success and complication rates were compared descriptively to results from other studies measuring DAP. No statistical inference testing was performed.

\section{Results}

The mean screening time measured by the DAP-meter was $53.8 \mathrm{~s}$ (range 1-320 s, median $33.0 \mathrm{~s}$ ). The mean DAP was $11.4 \mathrm{cGy} \mathrm{cm}^{2}$ (range $1-145 \mathrm{cGy} \mathrm{cm}^{2}$, median $5.45 \mathrm{cGy} \mathrm{cm}^{2}$ ). There was one perforation, in a 1-year-old boy who required surgical revision. In another child the screening time was $320 \mathrm{~s}$, which exceeded the screening time in the others by far. The reason was that the performing physicians thought they had not achieved appropriate repositioning of the ileum and continued the procedure over a prolonged period. However, surgical evaluation after pneumatic reduction could not confirm an intussusception, suggesting that the pneumatic reduction was successful. All other pneumatic reductions were successful and without complications. We did not find an association between the children's age and the mean DAP.

We found a limited number of studies that presented results on radiation exposure in children who received pneumatic or hydrostatic reduction of intussusception under fluoroscopy, and all these studies $[3,5,6]$ were published more than a decade ago.

Table 1 shows the results of our study in comparison with extracted data from the other three articles published on the topic. Although success and complication rates do not differ

Table 1 Comparison of radiation exposure and outcomes between our study and others

\begin{tabular}{|c|c|c|c|c|}
\hline & Our study & $\begin{array}{l}\text { Heenan et al. } \\
(2000)[3]\end{array}$ & $\begin{array}{l}\text { Karlsson et al. } \\
\text { (1994) [5] }\end{array}$ & Thomas et al. (1993) [6] \\
\hline Number of patients & 45 & 143 & 45 & 55 \\
\hline Number of reduction procedures & 48 & 153 & 45 & 58 \\
\hline Ultrasound used before fluoroscopy-guided reduction & Yes & Yes & No & No \\
\hline Type of reduction & Pneumatic & Pneumatic & Hydrostatic & 37 pneumatic; 21 hydrostatic \\
\hline Fluoroscopic monitoring & Pulsed & Non-pulsed & Non-pulsed & Non-pulsed \\
\hline Diagnostic tools used other than fluoroscopy & US & X-ray, US & X-ray & X-ray \\
\hline Mean screening time in seconds (range) & $53.8(1-320)$ & $354.0(15-1,356)$ & NA & NA \\
\hline Median screening time in seconds (range) & $33.0(1-320)$ & NA & $270.0(66-1,992)$ & $\begin{array}{l}66.0 \text { pneumatic; } 103.0 \text { hydrostatic } \\
\text { (ranges: NA) }\end{array}$ \\
\hline 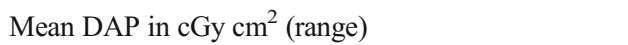 & $11.4(1-145)$ & $366.5(30-1,356)$ & NA & NA \\
\hline Median DAP in cGy $\mathrm{cm}^{2}$ (range) & $5.45(1-145)$ & NA & $\mathrm{NA}^{1}$ & $\begin{array}{l}400.0 \text { pneumatic; } 600.0 \text { hydrostatic } \\
\text { (ranges: NA) }\end{array}$ \\
\hline Success rate & $46 / 48(95.8 \%)$ & $117 / 153(76.5 \%)$ & $42 / 45(93.3 \%)$ & $46 / 58(79.3 \%)$ \\
\hline Complications & 1 perforation & 1 perforation & NA & 2 perforations \\
\hline
\end{tabular}

NA no data/information available

${ }^{1}$ DAP not given in this paper, instead mean absorbed dose (mGy) was given with a median 0.94 and range 0.16-9.4; energy imparted (mJ) was given with a median 10.8 and range $1.1-84.1$ 
substantially among studies, there is a huge difference in fluoroscopy screening times and radiation exposures. Our fluoroscopic screening times are about one-sixth of those found in Heenan et al. [3] and Karlsson et al. [5].

\section{Discussion}

The pneumatic reduction technique under fluoroscopy has gained wide acceptance because it is easy to perform, quick and clean [1, 7-10]. Success rates of reduction have been reported at $73-95 \%$ in earlier reports and at $74 \%$ on the average in more recent reports [2]. A theoretical dose-saving advantage of pneumatic reduction may be that air in the bowel absorbs less X-rays compared to contrast media. Thereby the dose rate in the measuring chambers of automatic exposure control chain is enormously lowered [11].

But air enema reduction requires precise control of the pressure and a thorough knowledge of the technique and potential complications. Regarding the issue of radiation exposure, an alternative to fluoroscopy is to use sonography not only for establishing the diagnosis but also for monitoring the reduction procedure. But it has been assumed that even greater experience is needed when using US guidance [1]. We, as an academic teaching hospital in which many residents are trained, decided to continue with pneumatic reduction under fluoroscopic guidance even after introduction of US-guided hydrostatic reduction in the 1980s [12]. Direct comparison of reduction using fluoroscopy versus US has not been studied [5], so there is no evidence that US can replace fluoroscopy as monitoring tool in intussusception reduction.

We found a limited number of studies that presented results on radiation exposure to children who received pneumatic or hydrostatic reduction of intussusception under fluoroscopy. Early research focused less on radiation dose than on the relative merits of pneumatic versus hydrostatic reduction techniques under fluoroscopic guidance [13].

Nevertheless, screening times and dose area products were considerably lower in our population than those reported in previous studies $[3,5,6]$. One reason for this may be that in the studies of Karlsson et al. [5] and Thomas et al. [6] diagnosis and reduction were performed with fluoroscopic guidance, whereas in Heenan et al. [3] and in our study diagnosis was performed with sonography, which decreases radiation time significantly. Mean screening time was $354 \mathrm{~s}$ (range 15-1,356 s) in the Heenan et al. [3] study, while it was only $53.8 \mathrm{~s}$ (range 1-320 s) in our study. Lowfrequency pulsed fluoroscopy and other modern dosesaving elements as well as our radiation risk training may account for this observation.

However, the indication of screening times with low-pulse technology may not be very reliable, especially if the screening is very short, because not all pulses are detected. Therefore the lower boundary of the given range of screening times may be a bit higher. Other limitations of our study were the retrospective design and the low number of performed reductions, which limited the generalizability of our findings. Further, a direct comparison between hospitals can be problematic. For example, tertiary and quaternary hospitals might admit more children with long-standing intussusception, and because these are more difficult to reduce the screening times could be longer at such institutions.

\section{Conclusion}

Our results in terms of radiation exposure are more favorable than those reported in decades past.

Conflicts of interest None

\section{References}

1. Daneman A, Alton DJ (1996) Intussusception. Issues and controversies related to diagnosis and reduction. Radiol Clin North Am 34: $743-756$

2. Applegate KE (2009) Intussusception in children: evidence-based diagnosis and treatment. Pediatr Radiol 39:S140-S143

3. Heenan SD, Kyriou J, Fitzgerald M et al (2000) Effective dose at pneumatic reduction of paediatric intussusception. Clin Radiol 55: $811-816$

4. Ilivitzki A, Shtark Glozman L, Arish K et al (2012) Deep sedation during pneumatic reduction of intussusception. Pediatr Radiol 42: $562-565$

5. Karlsson A, Schuwert P, Mortensson W (1994) Radiation exposure to children in diagnosing and at hydrostatic reduction of intussusception. Acta Radiol 35:296-299

6. Thomas RD, Fairhurst JJ, Roberts PJ (1993) Effective dose during screening monitored intussusception reduction. Clin Radiol 48: 189-191

7. Meyer JS, Dangman BC, Buonomo C et al (1993) Air and liquid contrast agents in the management of intussusception: a controlled, randomized trial. Radiology 188:507-511

8. Guo JZ, Ma XY, Zhou QH (1986) Results of air pressure enema reduction of intussusception: 6,396 cases in 13 years. J Pediatr Surg 21:1201-1203

9. Eshel G, Barr J, Heyman E et al (1997) Intussusception: a 9-year survey (1986-1995). J Pediatr Gastroenterol Nutr 24:253-256

10. Daneman A, Alton DJ, Ein S et al (1995) Perforation during attempted intussusception reduction in children - a comparison of perforation with barium and air. Pediatr Radiol 25:81-88

11. Persliden J, Schuwert P, Mortensson W (1996) Comparison of absorbed radiation doses in barium and air enema reduction of intussusception: a phantom study. Pediatr Radiol 26:329-332

12. Wang GD, Liu SJ (1988) Enema reduction of intussusception by hydrostatic pressure under ultrasound guidance: a report of 377 cases. J Pediatr Surg 23:814-818

13. Daneman A, Navarro O (2004) Intussusception. Part 2: an update on the evolution of management. Pediatr Radiol 34:97-108 